\title{
Mapping mania symptoms based on focal brain damage
}

\author{
Gonçalo Cotovio, ${ }^{1,2,3}$ Daniel Talmasov, ${ }^{4,5}$ J. Bernardo Barahona-Corrêa, ${ }^{1,2,3}$ Joey Hsu, ${ }^{6,7}$ Suhan Senova, ${ }^{1,8,9,10,11}$ Ricardo Ribeiro, ${ }^{1}$ \\ Louis Soussand, ${ }^{6,7}$ Ana Velosa, ${ }^{2}$ Vera Cruz e Silva, ${ }^{12}$ Natalia Rost, ${ }^{13}$ Ona Wu, ${ }^{14}$ Alexander L. Cohen,,${ }^{6,715}$ Albino J. Oliveira-Maia, $, 1,2,3$ \\ and Michael D. Fox $6,7,14,16,17$ \\ 'Champalimaud Research and Clinical Centre, Champalimaud Centre for the Unknown, Lisbon, Portugal. Department of Psychiatry and Mental Health, Centro Hospitalar de Lisboa Ocidental, Lisbon, \\ Portugal. ${ }^{3}$ NOVA Medical School | Faculdade de Ciências Médicas, Universidade Nova de Lisboa, Lisbon, Portugal. Department of Psychiatry, Brigham and Women's Hospital, Harvard Medical School, \\ Boston, Massachusetts, USA. ${ }^{5}$ Department of Neurology, New York University School of Medicine, New York, New York, USA. ${ }^{6}$ Berenson-Allen Center for Non-Invasive Brain Stimulation and ${ }^{7}$ Division of \\ Cognitive Neurology, Department of Neurology, Beth Israel Deaconess Medical Center, Harvard Medical School, Boston, Massachusetts, USA. ${ }^{8}$ Neurosurgery Department and ${ }^{9}$ PePsy Department, Groupe \\ Henri-Mondor Albert-Chenevier, Assistance Publique-Hôpitaux de Paris (APHP), Créteil, France. ${ }^{10}$ Equipe 14, U955 INSERM, Institut Mondor de Recherche Biomedicale and ${ }^{11}$ Faculté de Médecine, Université \\ Paris Est, Créteil, France. ${ }^{12}$ Department of Neuroradiology, Centro Hospitalar de Lisboa Ocidental, Lisbon, Portugal. ${ }^{13}$. Philip Kistler Stroke Research Center, Department of Neurology and ${ }^{14}$ Athinoula A. \\ Martinos Centre for Biomedical Imaging, Department of Radiology, Massachusetts General Hospital, ${ }^{15}$ Department of Neurology, Boston Children's Hospital, and ${ }^{16}$ Department of Neurology, Massachusetts \\ Ceneral Hospital, Harvard Medical School, Boston, Massachusetts, USA. ${ }^{17}$ Center for Brain Circuit Therapeutics, Department of Neurology, Department of Psychiatry, Department of Neurosurgery, and \\ Department of Radiology, Brigham and Women's Hospital, Harvard Medical School, Boston, Massachusetts, USA.
}

BACKGROUND. Although mania is characteristic of bipolar disorder, it can also occur following focal brain damage. Such cases may provide unique insight into brain regions responsible for mania symptoms and identify therapeutic targets.

\begin{abstract}
METHODS. Lesion locations associated with mania were identified using a systematic literature search $(n=41)$ and mapped onto a common brain atlas. The network of brain regions functionally connected to each lesion location was computed using normative human connectome data (resting-state functional MRI, $n=1000$ ) and contrasted with those obtained from lesion locations not associated with mania $(n=79)$. Reproducibility was assessed using independent cohorts of mania lesions derived from clinical chart review $(n=15)$ and of control lesions $(n=490)$. Results were compared with brain stimulation sites previously reported to induce or relieve mania symptoms.
\end{abstract}

RESULTS. Lesion locations associated with mania were heterogeneous and no single brain region was lesioned in all, or even most, cases. However, these lesion locations showed a unique pattern of functional connectivity to the right orbitofrontal cortex, right inferior temporal gyrus, and right frontal pole. This connectivity profile was reproducible across independent lesion cohorts and aligned with the effects of therapeutic brain stimulation on mania symptoms.

CONCLUSION. Brain lesions associated with mania are characterized by a specific pattern of brain connectivity that lends insight into localization of mania symptoms and potential therapeutic targets.

FUNDING. Fundação para a Ciência e Tecnologia (FCT), Harvard Medical School DuPont-Warren Fellowship, Portuguese national funds from FCT and Fundo Europeu de Desenvolvimento Regional, Child Neurology Foundation Shields Research, Sidney R. Baer, Jr. Foundation, Nancy Lurie Marks Foundation, Mather's Foundation, and the NIH.

\section{Introduction}

Patients presenting with psychiatric symptoms after focal brain damage challenge the boundaries between neurology and psy-

Authorship note: GC and DT are equally contributing first authors. AJOM and MDF are equally contributing senior authors.

Conflict of interest: AJOM is recipient of a grant from Schuhfried $\mathrm{GmBH}$ for norming and validation of cognitive tests, and is national coordinator for Portugal of a noninterventional study (EDMS-ERI-143085581, 4.0) to characterize a Treatment-Resistant Depression Cohort in Europe, sponsored by Janssen-Cilag, Ltd., and of a trial of psilocybin therapy for treatment-resistant depression, sponsored by Compass Pathways, Ltd. (EudraCT number 2017-003288-36). JBBC received honoraria in 2018 as member of the local Advisory Board for Trevicta from Janssen-Cilag Ltd. Copyright: ( 2020, American Society for Clinical Investigation. Submitted: January 2, 2020; Accepted: June 24, 2020; Published: August 24, 2020. Reference information: J Clin Invest. 2020;130(10):5209-5222. https://doi.org/10.1172/JCI136096. chiatry $(1,2)$. Such cases are of interest as they can provide causal links between brain regions or circuits and specific psychiatric symptoms, and lead to identification of therapeutic targets (3, 4). However, judging whether new-onset psychiatric symptoms are attributable to a structural brain lesion can be complicated by incidental findings on neuroimaging (5), potential delays between the lesion and the resulting symptoms (6), and because the same symptoms can be caused by damage to multiple different brain regions (7).

Mania is a paradigmatic example. It is characteristic of bipolar I disorder, but has been reported following focal brain lesions for nearly a century (8). In seminal work by Starkstein and Robinson, lesions associated with mania were found to be more common in the right hemisphere, but occurred in numerous different brain regions (9-19). These authors hypothesized that lesions causing mania may map to a limbic circuit involving 


\section{A}
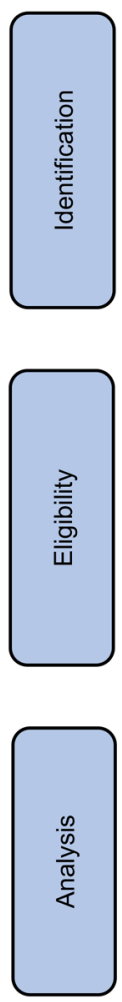

B
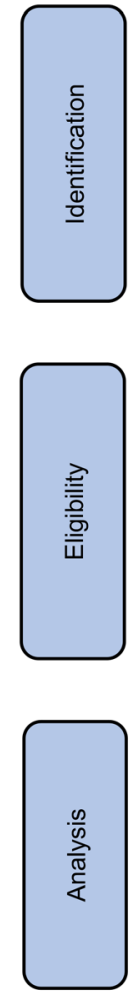

the orbitofrontal cortex, basotemporal cortex, and basal ganglia (9-19). A recently developed technique, termed lesion network mapping, is well suited to test this hypothesis $(3,20)$. Rather than focus on lesion locations alone, this approach examines the network of regions functionally connected to each lesion, using resting-state functional connectivity $(21,22)$ from a

\section{Figure 1. Lesional mania} cohorts CONSORT diagrams.

Forty-one and 15 lesional mania cases were included in our literature $(\mathbf{A})$ and clinical (B) lesional mania cohorts, respectively.
Excluded from the analysis due to due to poorly defined brain lesion limits $(n=8)$

- Traumatic brain injury $(n=5)$

- Other $(n=1)$
Excluded $(n=3)$

- Insufficient clinical information $(n=1)$

- Did not meet inclusion criteria $(n=2)$

- Mania secondary to other causes $(n=1)$

- Diagnosis revised to Paranoid Schizophrenia $(n=1)$

Excluded from the analysis due to poorly defined brain lesion limits $(n=2)$

- Traumatic brain injury $(n=1)$

- Brain infection $(n=1)$ large database of healthy volunteers $(n=1000)(23,24)$. By mapping symptoms to brain networks, rather than single brain regions, this technique has lent insight into mechanisms underlying a variety of neuropsychiatric symptoms $(4,20,25$, 26). Here, it was applied to 2 independent cohorts of lesions associated with mania. 


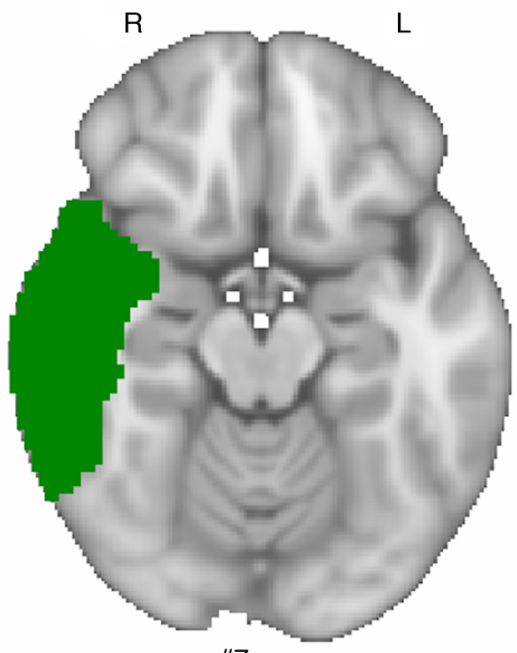

\#7

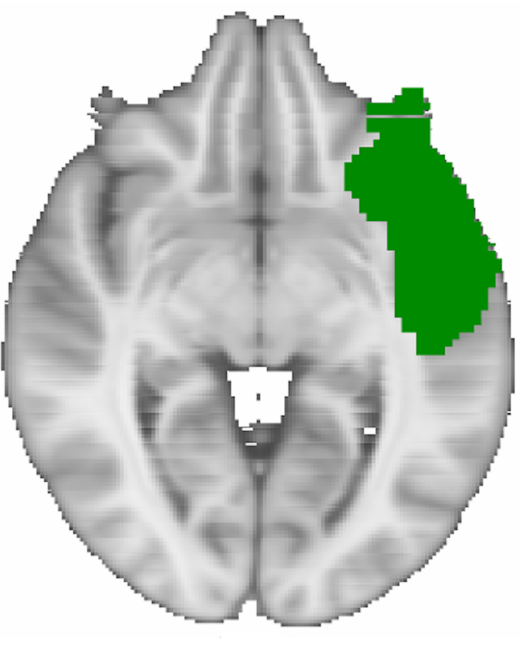

\#24

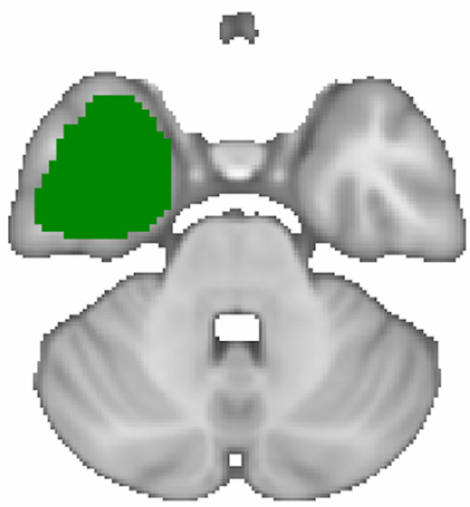

\#8

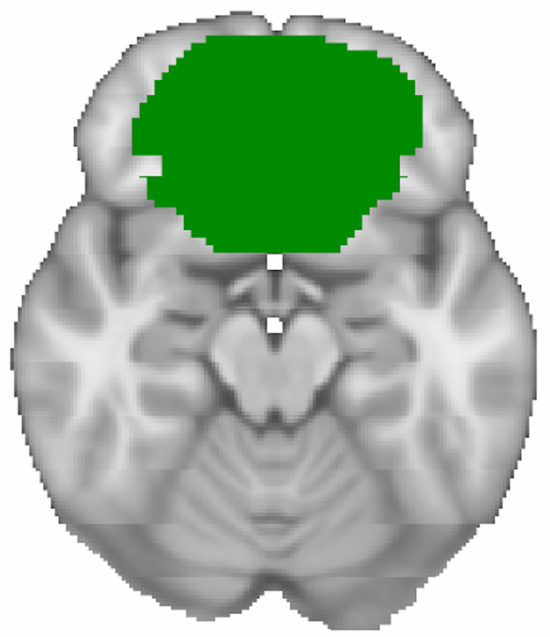

\#32

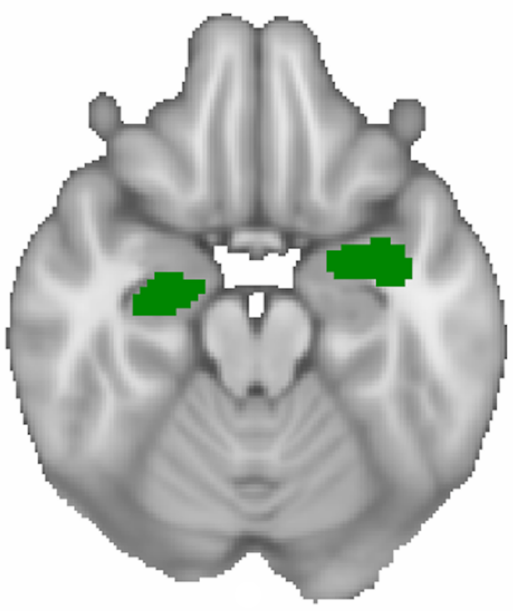

\#12

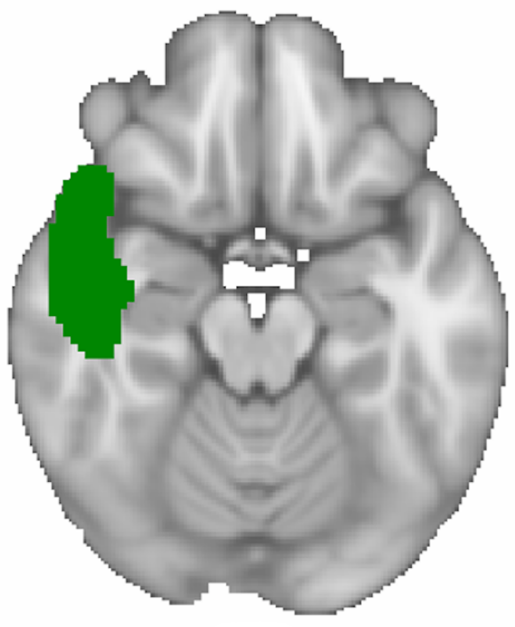

\#39

Figure 2. Lesions associated with mania occur in multiple different brain locations. Six representative lesions (green) selected from 41 literature cases demonstrate heterogeneity in lesion location.

\section{Results}

Deriving a mania network from brain lesions in the literature. Our systematic literature search identified 41 brain lesions associated with mania and fulfilling inclusion and exclusion criteria (Figure 1A, literature cohort. See supplemental material for details, available online with this article; https://doi.org/10.1172/ JCI136096DS1). The mean age of patients was $54.9 \pm 17.7$ years and the most common symptoms were mood elation/irritability, increased energy, increase in goal-directed activity, and psychomotor agitation. Pressured speech and decreased need for sleep were also very common. The time between lesion and symptom onset ranged from hours to years, with a median time of 3 years (Supplemental Table 1). When these lesion locations were mapped to a common brain atlas, they were found to be neuroanatomically diverse, involving a wide range of cortical and subcortical areas (Figure 2 and Supplemental Figure 1). No single brain region was lesioned in all or even most cases.

Given this heterogeneity, we next tested whether these lesion locations shared a specific pattern of brain connectivity (Figure
3). Similar to prior work $(3,20)$, we computed functional connectivity between each lesion location and the rest of the brain using resting-state functional MRI (rs-fMRI) data from a large normative cohort $(n=1000)(23,24)$. The connectivity pattern of mania lesions was then compared with that of control lesions also derived from the literature, but not associated with mania symptoms $(n=$ $79)(20,27,28)$. We found significant differences in lesional connectivity between the 2 groups, with peaks in the right orbitofrontal cortex and right temporal cortex (Figure 3 and Supplemental Figure 2). We refer to this map as a "mania lesion network," with lesion locations associated with mania intersecting positive nodes in this network map (Figure 3F), and control lesions not associated with mania intersecting neutral or negative nodes (not shown).

Reliability, validation, reproducibility, and robustness. To asses reliability of tracing methods, all 41 literature-based lesions were retraced by a different researcher, who was blind to the original lesion tracings. Lesion masks produced by the 2 tracers were very similar. The median distance between the center of gravity of tracings for the same lesion ( $8 \mathrm{~mm}, 3$ voxels) was significantly smaller 

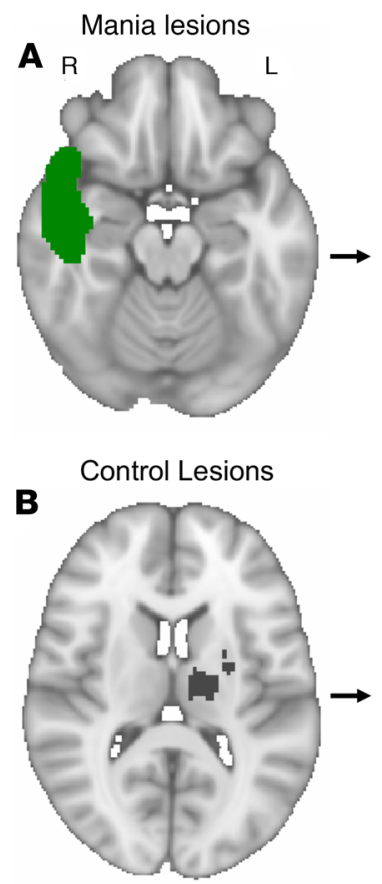

Functional connectivity
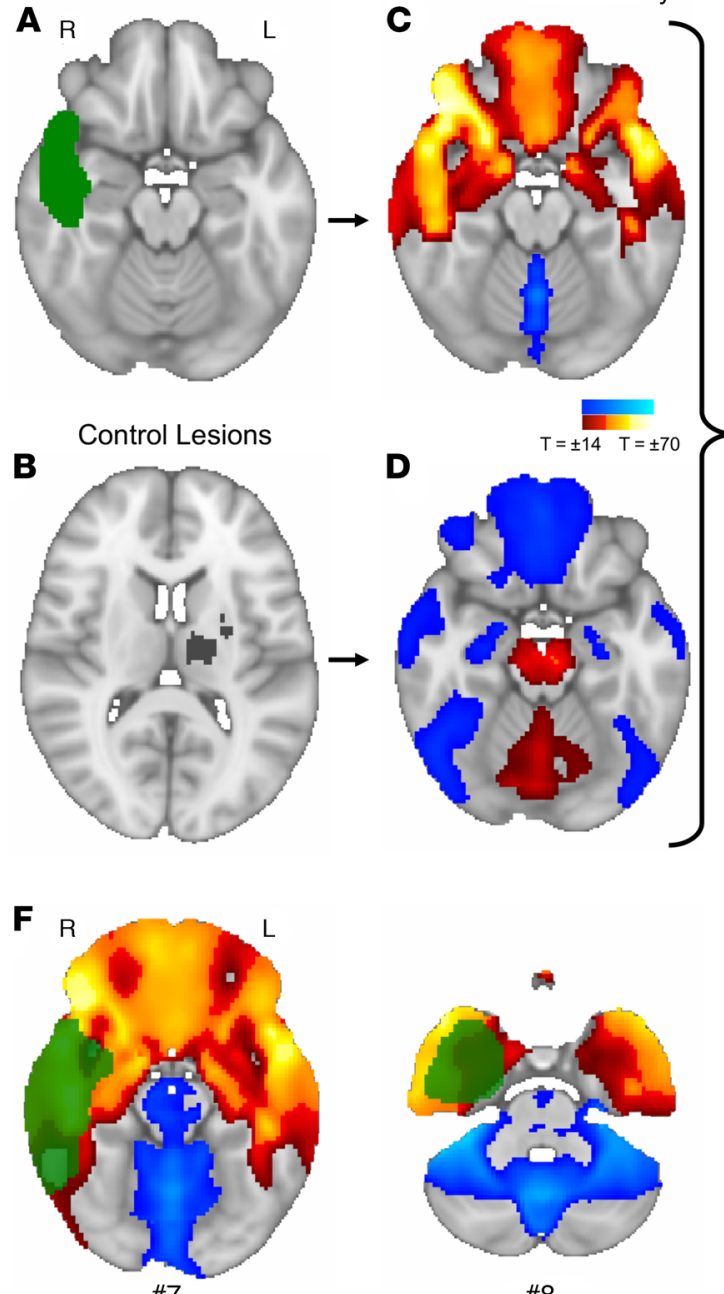

\#7

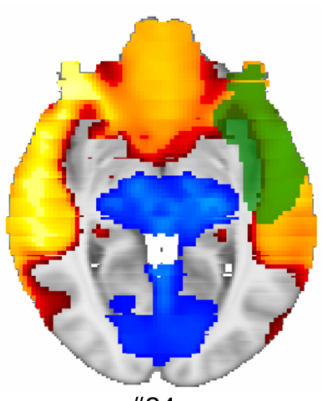

\#24

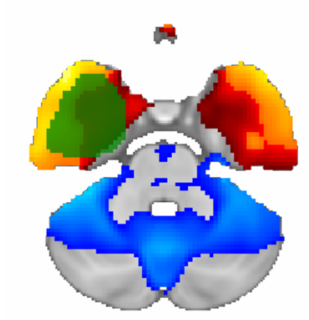

\#8

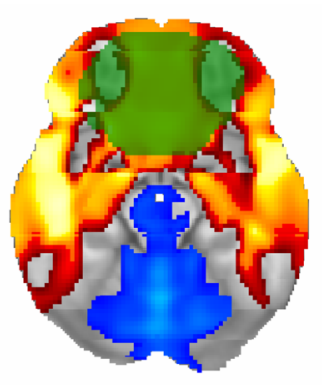

\#32
E

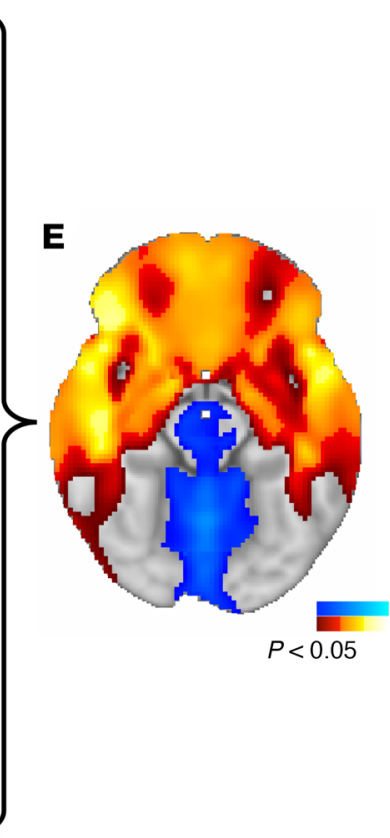

Mania vs. control

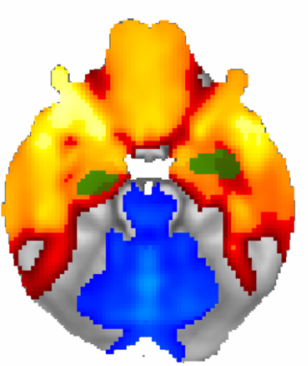

\#12

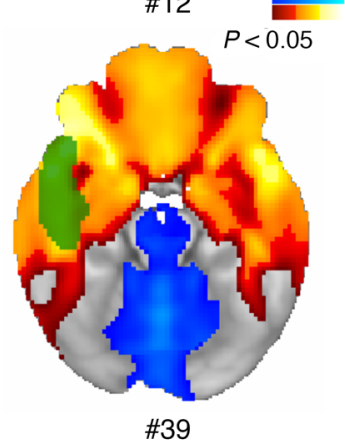

Figure 3. Deriving a mania network from brain lesions in the literature. The location of brain damage associated with mania (A) or with other unrelated symptoms (B) was identified based on published images and mapped to the MNI standard brain atlas. (C and $\mathbf{D}$ ) Brain regions functionally connected to each lesion location were identified based on a large normative resting-state functional connectivity database connectivity database $(N=1000)$. Results were combined across the 1000 subjects to generate a statistical T map for each lesion. Statistically significant differences in functional connectivity between mania lesions $(n=41)$ and control lesions ( $n=79$ ) define a "mania lesion network map." In this map (E), regions more connected to mania lesions are shown in warm colors, while regions more connected to control regions are shown in cool colors. (F) Lesion locations from literature mania cases (green) overlap positive nodes in the network. Maps in $\mathbf{E}$ and $\mathbf{F}$ were obtained using a voxel-wise permutation-based 2-sample $t$ test performed within FSL PALM (2000 permutations) and are displayed at an FWE-corrected level of $P<0.05$. than that between different lesions $(51 \mathrm{~mm}, 26$ voxels, $P<0.0001)$. When our mania lesion network was recomputed using lesion masks from the independent tracer, results were nearly identical to the original network (spatial correlation of 0.96). For additional details see Supplemental Figure 3.

To validate the mania network derived from literature-based lesions, we identified a second cohort of lesion-induced mania cases based on clinical chart review (Figure 1B). These 15 cases were of similar age (54.9 \pm 17.0 years) and had similar mania symptoms compared with our original literature cohort (Supplemental Table 2). However, the lesions were located in different brain areas (Supplemental Figure 1). For example, the region of maximum lesion overlap in our literature cohort only intersected 1 lesion in our clinical cohort, while the region of maximum overlap in our clinical cohort did not intersect any lesion in our literature cohort (Supplemental Figure 1). However, lesion locations from the clinical cohort did intersect the mania lesion network map derived from the literature cohort (Figure 4, A and B). Lesion locations from the clinical mania cohort demonstrated significantly more overlap with positive nodes of the literature-based mania lesion network map when compared with more than 490 clinical control lesions not associated with mania $(P<0.05)$.

To assess reproducibility, we computed a new mania lesion network map by comparing the connectivity of clinical mania lesions to that of clinical control lesions (Figure 4C). This clinical mania lesion network map aligned well with the mania lesion network derived from the literature cohorts (Figure 4B). A conjunction analysis identified connections significantly associated with mania across both independent cohorts (Figure 4D), while a combined analysis across both data sets identified connections most strongly associated with mania, even at a rigorous statistical threshold of $\alpha$ less than $1.0 \times 10^{-4}$ (Figure 4E; see Supplemental Figures 2 and 4 for additional detail). The most significant connections in this combined map were the right orbitofrontal cortex (Montreal Neurologic Institute [MNI] 42, 42, -20), right lateral temporal cortex (MNI 50, -26, -16), and right frontal polar cortex (MNI 12, 54, -14) (Figure 4E and Supplemental Table 3).

Our combined mania lesion network was robust in controlling for various confounds. Specifically, it remained similar when controlling for lesion size (Figure 5A) when using different sets of control lesions, including lesions associated 
A

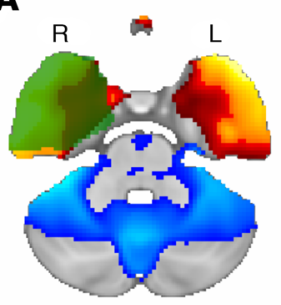

\#3

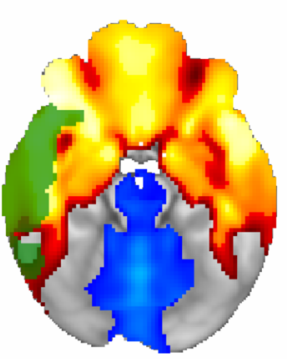

\#9

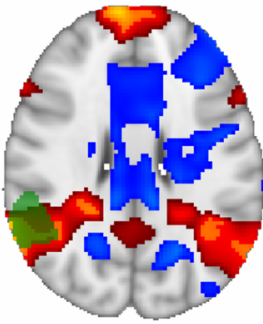

\#4

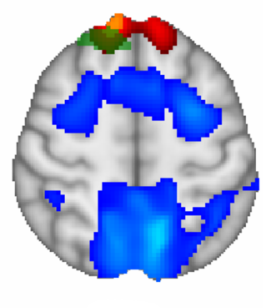

\#13
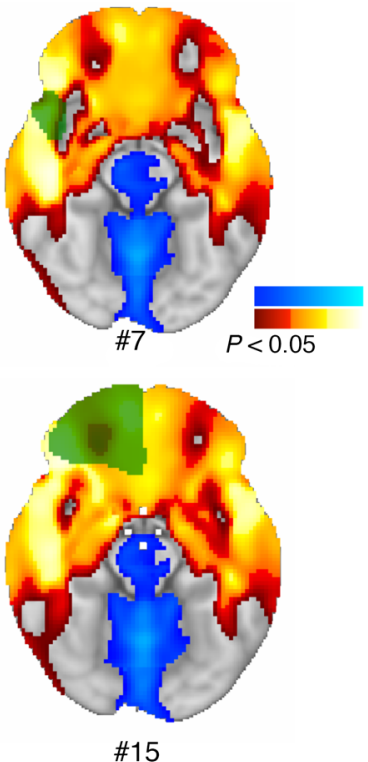

B
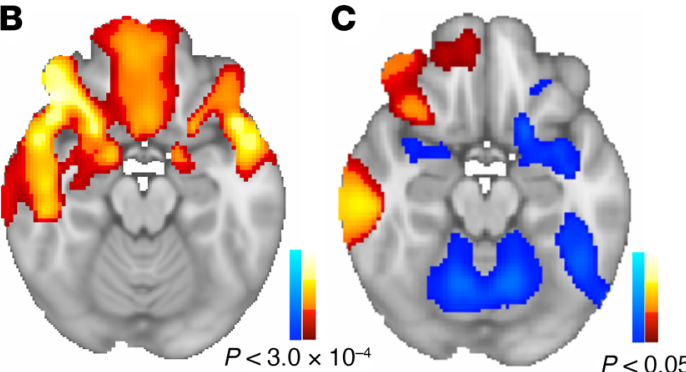

D

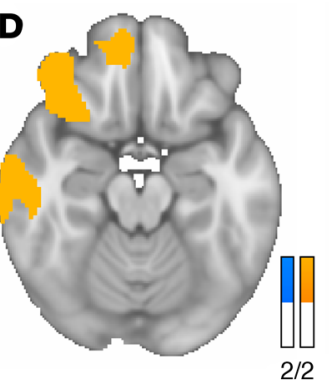

$\mathbf{E}$

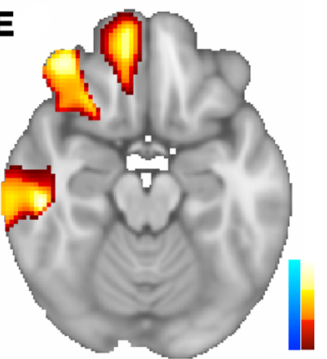

$2 / 2$

$P<1.0 \times 10^{-4}$

Figure 4. Validation and replication of lesion mania network with an independent data set. (A) Lesion locations from a clinical mania lesion cohort (green, 6 of 15 lesions shown) intersect positive nodes in the mania lesion network map derived from literature cohorts. The mania lesion network map derived from literature cohorts ( $n=41$ vs. 79 mania vs. control lesions) (B) was reproducible when using data from clinical cohorts ( $n=15$ vs. $n=490$ mania vs. control lesions) (C). A conjunction analysis shows significant regions identified across both mania lesion network maps (overlap of B and $\mathbf{C}$ ) (D) and a single mania lesion network map combining both literature and clinical cohorts shows regions that are strongly associated with mania when using a high statistical threshold ( $n=56$ vs. $n=569$ mania vs. control lesions) (E). Connectivity maps were obtained using a voxel-wise permutation-based 2 -sample $t$ test performed within FSL PALM (2000 permutations). Connectivity maps in $\mathbf{A}$ and $\mathbf{C}$ are displayed at an FWE-corrected level of $P<0.05$. Connectivity maps in $\mathbf{B}$ and $\mathbf{E}$ are displayed at more stringent FWE-corrected levels $\left(P<3.0 \times 10^{-4}\right.$ and $P<1.0 \times 10^{-4}$, respectively), in order to best show the peak regions of each map. For additional details on peak locations see Supplemental Table 3 and Supplemental Figure 5 . The map in D was obtained by binarizing B and C and computing the sum of their overlap. The maps in B-E are at MNI space $z=-18$. Regions more connected to mania lesions are shown in warm colors, while regions more connected to control regions are shown in cool colors.

with other psychiatric symptoms (Figure 5B), or when considering time between lesion and symptom onset in the analyses (Figure 5, $\mathrm{C}$ and $\mathrm{D}$ ). The network also remained similar when analyses were restricted to the subset of patients with all core mania symptoms (Figure 5E), patients with absent personal or family history of neuropsychiatric syndromes (Figure $5 \mathrm{~F}$ ), those with acute ischemic stroke as the etiology for brain lesions (Figure $5 \mathrm{G}$ ), or those with all these restrictions combined (Figure $5 \mathrm{H}$ ).

Diagnostic relevance of the mania lesion network. To explore potential clinical applications of such a mania lesion network, it was used to analyze a diagnostically challenging case, published after our original literature search (2). In this case, new-onset mania symptoms were attributed to an acute stroke-induced lesion, in the presence of chronic brain lesions and prior mania-like symptoms. Based on intersection of the lesion locations from this case with our combined mania lesion network (Figure 6, A and B), we found that the acute brain lesion was unlikely to contribute to mania symptoms (1.3\% probability of falling within the mania lesion distribution, $67 \%$ probability of falling within the control lesion distribution). In contrast, 1 of the older lesions was a more likely contributor (46\% probability of falling within the mania distribution, $<1 \%$ probability of falling within the control distribution).

Relationship of the mania lesion network to lesion locations causing other symptoms. In an exploratory analysis, we tested whether lesion locations causing other neuropsychiatric symptoms inter- sected the combined mania lesion network (20, 25, 26, 29-31). We found that lesion locations associated with criminality and delusions intersected the mania network more than lesion locations associated with movement disorders or hallucinations $(P<$ 0.0001; Figure 6, C and D).

Therapeutic relevance of the mania lesion network. Finally, we explored whether the combined mania lesion network aligns with previously reported effects of brain stimulation on mania symptoms (Figure 7, A-F). Specifically, high-frequency repetitive transcranial magnetic stimulation (rTMS) of the right dorsolateral prefrontal cortex (DLPFC) appears to be beneficial for mania (32-35), but high-frequency stimulation of the left DLPFC, used as treatment for depression, may induce manic symptoms $(36,37)$. Consistent with these reports, we found that left and right DLPFC regions of interest (ROIs) encompassing these rTMS targets have opposite connectivity to the mania network map $(P<0.0001$; Figure 7, A and B). Moreover, rTMS treatment protocols targeting the right DLPFC using the 5 -cm rule $(34,35,38)$ reported greater improvement in mania symptoms than a treatment protocol targeting a more anterolateral location (39). Consistent with these reports, the $5-\mathrm{cm}$ target is more connected to our mania network map than the less effective anterolateral target $(P<0.0001$; Figure 7, $C$ and D). Given this preliminary evidence, we identified the peak positive connectivity site of the mania network map in the right DLPFC (MNI 20, 36, 52), and peak negative connectivity sites in the left DLPFC (MNI-20, 2, 64 and $-36,44,38$ ), for 

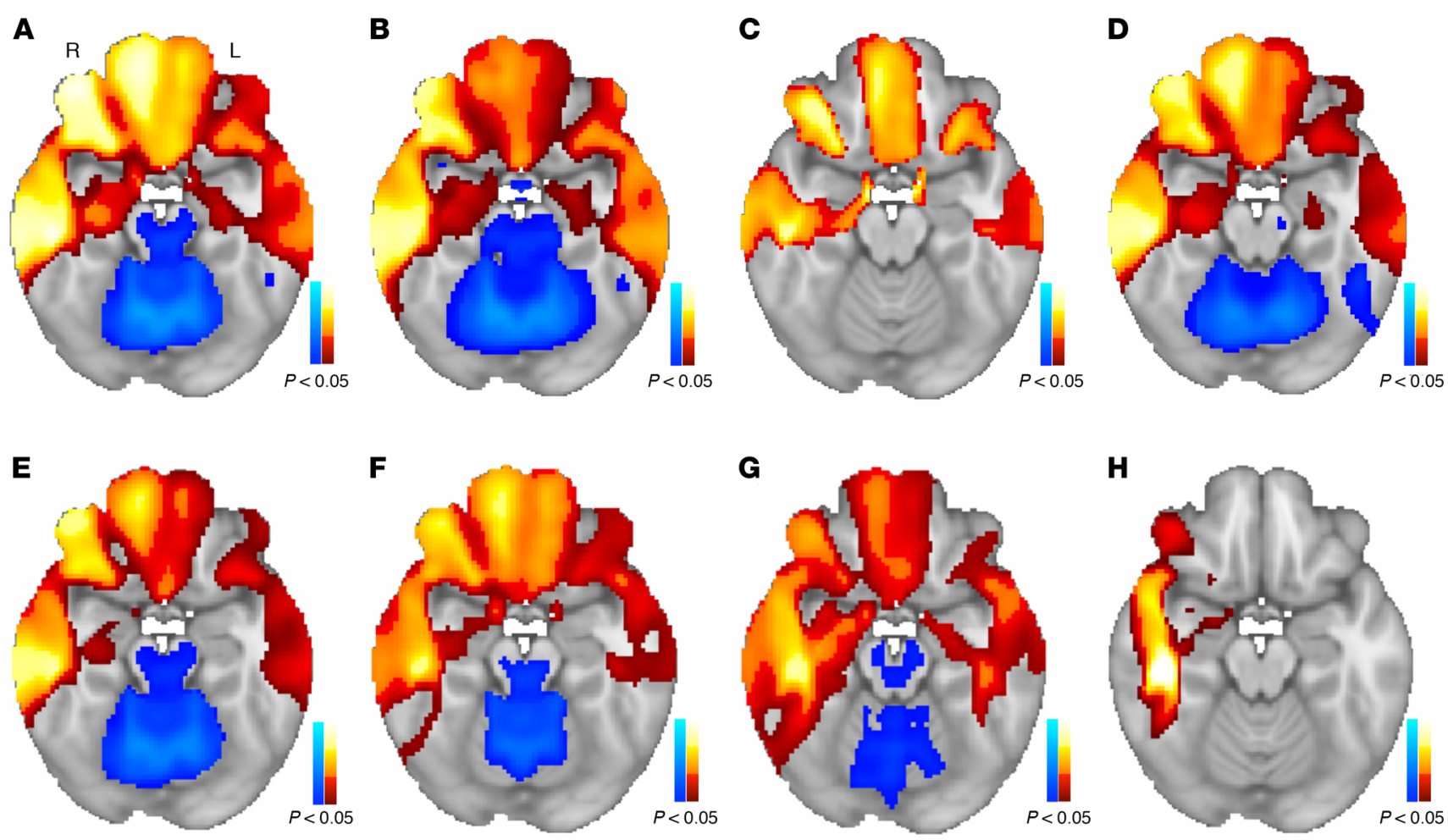

Figure 5. Stability of mania lesion network when controlling for various confounds. The combined mania lesion network, using data from literature and clinical cohorts, remained similar when it was recomputed controlling for lesion size ( $n=56$ vs. $n=569$ mania vs. control lesions) (A), when using a different set of control lesions ( $n=56$ vs. $n=409$ mania vs. control lesions; see Methods for details) (B), and when using a subset of mania cases with shorter temporal association between lesion and symptom onset ( $n=28$ vs. $n=569$ mania vs. control lesions) (C) or the remaining mania cases ( $n=28$ vs. $n=569$ mania vs. control lesions; see Methods for details) (D). The lesion network was also similar when restricting analyses to subsets of lesional mania cases presenting with all core mania symptoms as listed in DSM 5 ( $n=46$ vs. $n=569$ mania vs. control lesions) (E), with no personal or family history of relevant neuropsychiatric syndromes ( $n=39$ vs. $n=569$ mania vs. control lesions) (F), caused by ischemic stroke ( $n=23$ vs. $n=569$ mania vs. control lesions) (G), or accumulating all of the restrictions mentioned in E-C ( $n=19$ vs. $n=569$ mania vs. control lesions) (H). Connectivity maps were obtained using a voxel-wise permutationbased 2-sample $t$ test performed within FSL PALM (2000 permutations) and are displayed at an FWE-corrected level of $P<0.05$, at MNI space $z=-18$. Regions more connected to mania lesions are shown in warm colors, while regions more connected to control lesions are shown in cool colors.

potential future use in rTMS trials (for additional details on peak locations see Supplemental Figure 5).

In similar analyses, we explored whether the mania lesion network map aligns with reports of mania symptoms following deep brain stimulation (DBS). Specifically, mania has been reported following DBS of the ventral capsule/ventral striatum (VC/VS) for obsessive-compulsive disorder, but it does not occur in most patients (40). Consistent with our findings, the location of stimulation associated with the occurrence of mania (41-44) is more connected to the mania lesion network than the standard location of stimulation ( $P<0.0001$; Figure 7, E and F, and ref. 45). Similarly, mania is considered a rare complication of subthalamic nucleus (STN) DBS for Parkinson's disease (46), and stimulation sites that have been associated with mania (47-49) are more connected to our identified mania lesion network than the standard location of stimulation $(P<0.0001$; Supplemental Figure 6 and ref. 50).

\section{Discussion}

Why some lesions, but not others, are associated with mania has remained unclear since the association was first noted nearly a century ago (8). Despite heterogeneity in lesion location, we found that lesion locations associated with new-onset mania are characterized by connectivity to the right orbitofrontal cortex, right lateral temporal cortex, and right frontal polar cortex. This result was reproducible across independent lesion cohorts and aligns with reported effects of therapeutic brain stimulation on mania symptoms.

Our findings are based on lesion network mapping, a technique that has been successfully applied to hallucinations (20, 51 ), delusions (25), criminality (26), depression (4), and many other neuropsychiatric symptoms (3). In fact, our study is not the first to apply lesion network mapping to mania, with a prior study also concluding that mania lesions show a distinct pattern of brain connectivity (52). However, which connections are most important differ between the 2 studies, including the description of right-sided lateralization here. These differences are likely due to several methodological improvements in our study, including a larger mania cohort ( $n=56$ vs. 23$)$ (53), a larger connectome data set ( $n=1000$ vs. 40$)(26,54)$, and a larger number of control lesions ( $n=569$ vs. 23). Our 41 literature lesions include 21 of the 23 lesions from this prior study ( 1 case was excluded due to being on antidepressants [ref. 55] and another case was published after our literature search [ref. 56]). Our study also extends beyond prior work by investigating reproducibility in an independent mania lesion cohort $(N=15)$, 
A

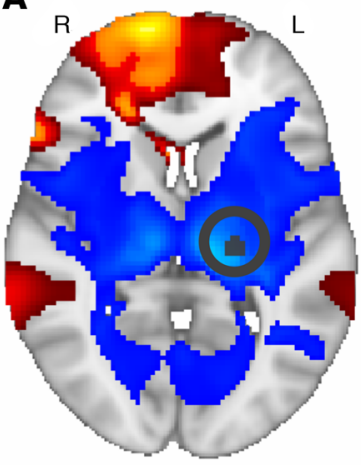

Acute lesion
B

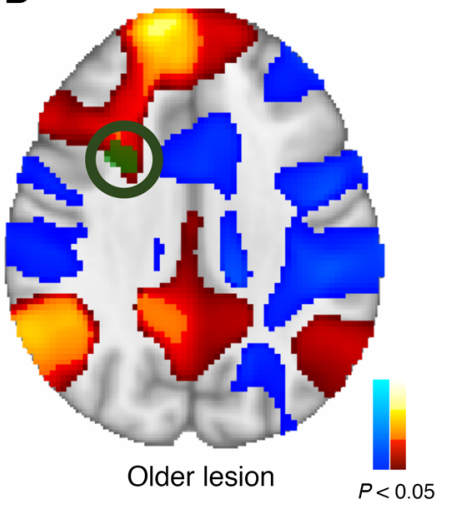

c

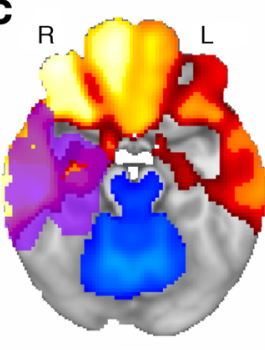

\#1

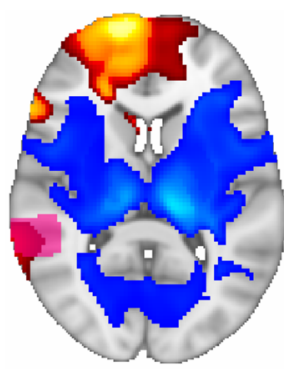

\#1
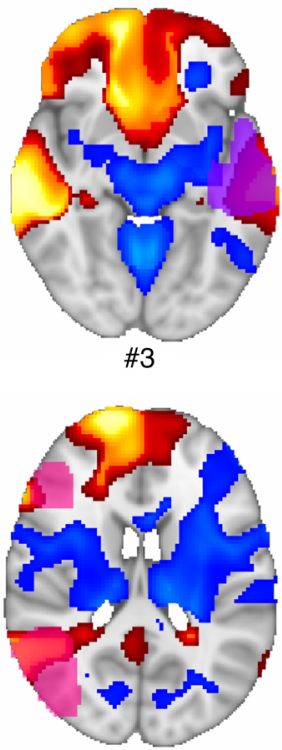

\#4
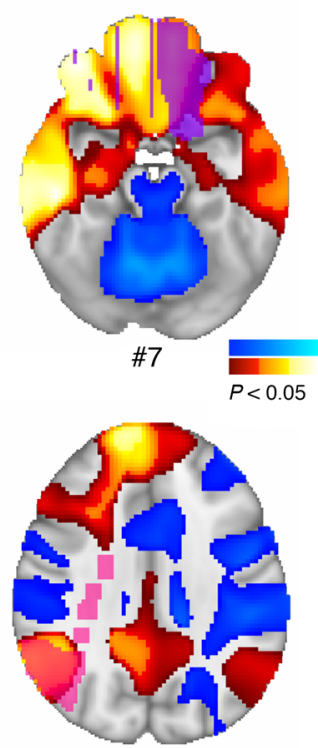

\#15

D

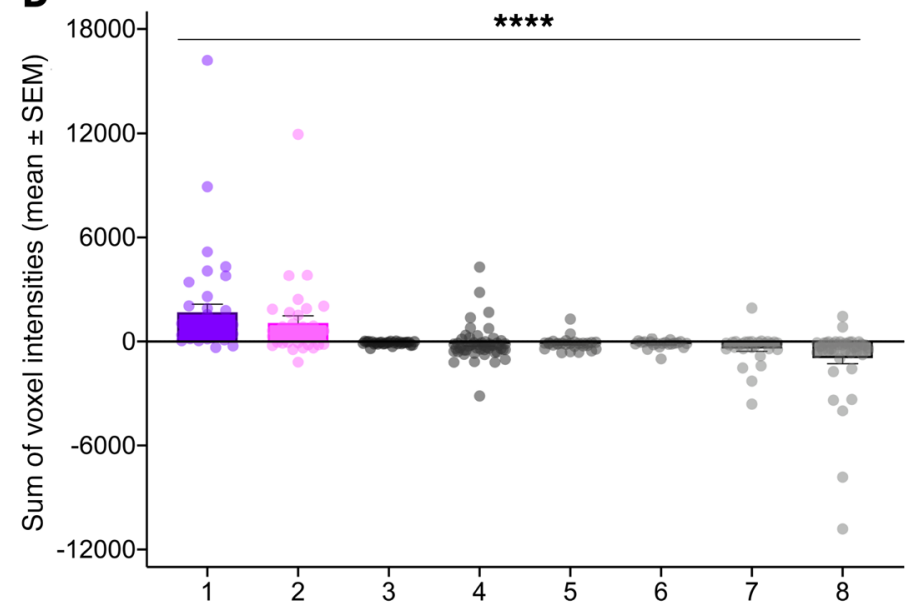

1. Criminality $(N=40)$

2. Delusions $(N=32)$

- 3. Hemichorea $(N=29)$

4. Alien limb $(N=53)$

5. Akinetic mutism $(N=28)$

7. Parkinsonism $(N=29)$

6. Auditory hallucinations $(N=21)$

8. Visual hallucinations $(N=42)$
Figure 6. Intersection of our mania lesion network with other lesion locations. (A) We explored a diagnostically challenging case of lesional mania (2), where mania symptoms were attributed to an acute infarct. This acute infarct intersected a negative node of our mania lesion network, making it an unlikely contributor to mania symptoms. (B) In contrast, a previous lesion intersected a positive node of our network, consistent with a history of mania-like symptoms predating the acute infarct. (C) In a separate analysis, lesion locations associated with other neuropsychiatric symptoms, such as criminality (purple) and delusions (pink), intersected our mania lesion network map. (D) This intersection differed significantly across different neuropsychiatric symptoms. The $y$ axis in $\mathbf{D}$ represents the sum of the voxel intensities in the mania lesion network map at the location of each lesion. Connectivity maps in A-C were obtained using a voxel-wise permutation-based 2-sample $t$ test performed within FSL PALM (2000 permutations) and were corrected for multiple comparisons using threshold-free cluster enhancement and displayed at an FWEcorrected level of $P<0.05$. Regions more connected to mania lesions are shown in warm colors, while regions more connected to control regions are shown in cool colors. ${ }^{* * *} P<0.0001$ in a general linear model controlling for lesion size.

and exploring diagnostic potential, relationship with lesion locations causing other neuropsychiatric symptoms, and therapeutic and iatrogenic relevance in TMS and DBS.

Our study found a clear right-sided lateralization of connections specific to mania lesions. This lateralization was present both in the literature and clinical cohorts when analyzed independently, but it was most obvious when combining the data sets and focusing on only the most significant findings (see Figure 4). This right lateralization is consistent with the valence hypothesis of mood laterality $(10,57)$ and prior right-lateralized findings in mania, including studies of lesion location $(58,59)$, lesion-induced hypoperfusion $(16,26,60)$, brain stimulation (32-37), and neuroimaging findings in primary bipolar disorder (60-72). More specifically, our results show that lesion locations associated with mania are connected to the right orbitofrontal cortex and right lateral temporal cortex, consistent with the longstanding hypothesis of Starkstein and Robinson (9-19).

Mania lesion network maps may be useful for determining whether a given lesion is related to mania symptoms, similar to prior work in delusions (73). Structural neuroimaging is increasingly acquired for workup of psychiatric symptoms, but incidental abnormalities are common (74). A recently published case report illustrates this diagnostic challenge for mania (2). In this case, a 61-year-old man presented to the emergency department with increased energy, irritability, euphoria, grandiosity, impulsive behavior, and decreased need for sleep. These manic symptoms had been present for over a month, but the lesion considered to be responsible for these symptoms was likely less than 5 days old based on brain imaging of the lesion (hyperintense in diffusion-weighted MRI and hypointense on apparent diffusion coefficient MRI) $(75,76)$. Exploratory use of our mania lesion network map suggested that an older lesion was a more likely contributor to the patient's mania symptoms than the acute lesion, which is consistent with the patient's history of psychiatric symptoms, predating the acute lesion 


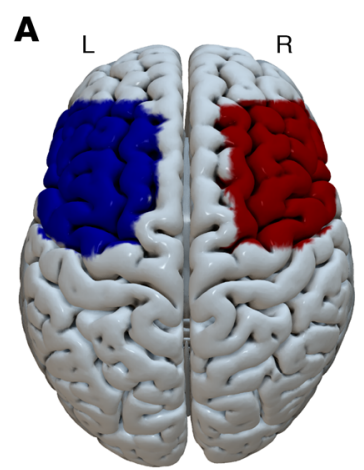

C rTMS targets for treatment of mania

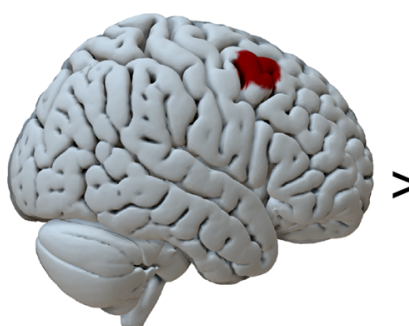

Right Average $5 \mathrm{~cm}$

D
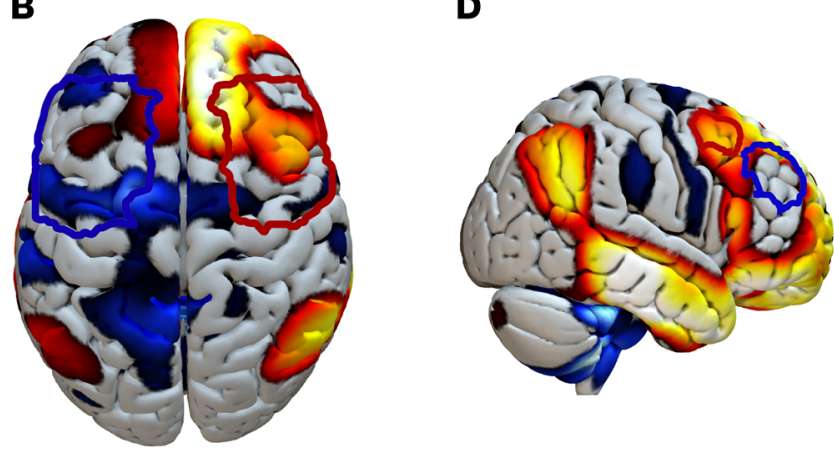

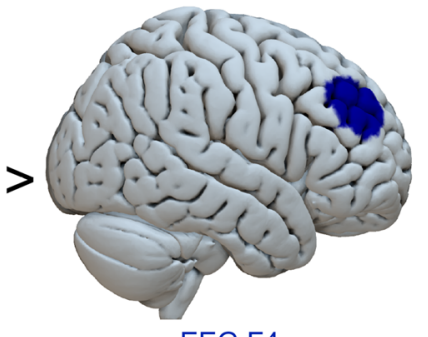

EEG F4

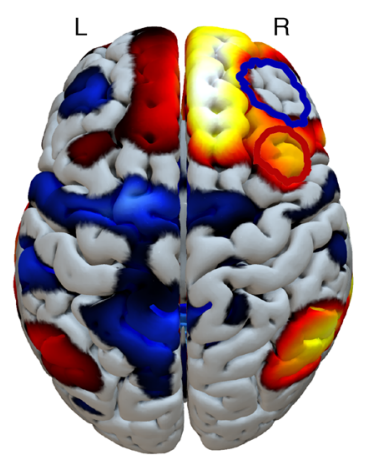

E

VCNS targets for DBS

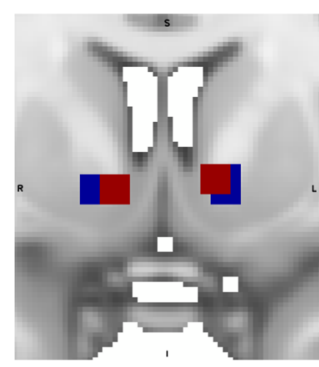

Mania-inducing targets
$\mathbf{F}$

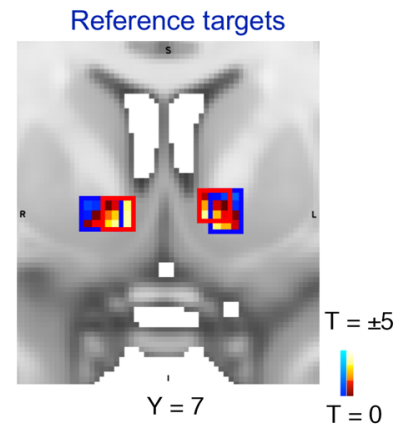

Figure 7. Potential therapeutic relevance of the mania lesion network. (A) High-frequency repetitive transcranial magnetic stimulation (rTMS) of the right dorsolateral prefrontal cortex (red region) has been reported to improve mania symptoms (32-35, 92), while high-frequency rTMS and anodal transcranial direct current stimulation of the left dorsolateral prefrontal cortex (blue region) can induce mania (36, 37, 93-97). (B) These stimulation sites overlay areas of mostly positive connectivity in the mania lesion network map (red outline), and mostly negative connectivity (blue outline). (C) rTMS trials targeting a brain region identified with the 5 -cm rule (red region) reported increased therapeutic efficacy for mania compared with trials targeting a region identified using the EEG F4 coordinate (blue region). (D) The more effective target intersects our mania lesion network (red outline), while the less effective target does not (blue outline). (E) Deep brain stimulation (DBS) sites reported to induce mania (red region) are slightly offset compared with the standard deep brain stimulation site in the ventral capsule/ventral striatum (VC/VS) (blue region). (F) The site reported to induce mania intersects our mania lesion network (red outline), while the standard site does not (blue outline). DBS sites in panels $\mathbf{E}$ and $\mathbf{F}$ are shown on a coronal brain slice (MNI space $y=7$ ). Connectivity maps in B, D, and F were obtained using a voxel-wise permutation-based 2-sample $t$ test performed within FSL PALM (2000 permutations). $\mathbf{B}$ and $\mathbf{D}$ are displayed at an FWE-corrected level of $P<0.05$. In these panels, regions more connected to mania lesions are shown in warm colors, while regions more connected to control regions are shown in cool colors.

(2). However, it is important to emphasize that the mania lesion network maps developed here are maps of probability, rather than maps of regions where lesions will always cause mania. Many patients with lesions in the red-yellow area of our map will not present with clinically apparent mania. Mania is relatively rare following focal brain damage $(<10 \%)$, compared with other symptoms such as depression (21\%-33\%) (77-80), which is consistent with observed differences in global prevalence between primary bipolar disorder and major depressive disorder $(81,82)$, and the fact that depression is a more common reaction to any new medical condition (83). It is also possible that patients with lesions falling within our mania network will experience mild or subclinical symptoms, as recently shown for lesion network maps of depression (4), memory (84), and facial recognition (85). Finally, there are likely other factors that play a role in lesion-induced mania besides lesion location, including genetic $(16,86)$ and environmental (87-89) factors. Additional work, using larger cohorts, will be needed to determine the diagnostic utility of mania lesion networks, including for prediction of subclinical mania symptoms.
While the mania lesion map developed here may be relevant to address diagnostic questions, it is worth noting that there is overlap with lesional neuropsychiatric syndromes with overlapping symptoms. In fact, an important and potentially novel aspect of the current study is the use of lesion network mapping to relate different lesion-induced symptoms to one another. We found that lesion locations associated with criminality (26) and delusions (25) showed strong intersection with the mania lesion network map, to a larger degree than lesion locations associated with other symptoms such as hallucinations. This is consistent with increased risk of criminal problems (90) and presence of delusions (91) in patients suffering from mania, as well as with a higher incidence of delusions compared with hallucinations in these patients (91). Lesion network mapping may thus prove useful for understanding the neuroanatomical relationship between different comorbid neuropsychiatric symptoms, extending well beyond mania.

Similar to lesional syndromes, mania is considered a rare complication of DBS $(40,46)$. Our mania lesion network results are consistent with observed differences in mania resulting from different DBS electrode locations, for both the VC/VS and STN 
targets. Although speculative, our results may have implications for focal brain stimulation treatment of mania. High-frequency rTMS to the right DLPFC may be beneficial in mania $(32-35,92)$, with some right prefrontal rTMS sites appearing to be more effective than others $(34,35,38,39)$. On the other hand, high-frequency rTMS and anodal transcranial direct current stimulation (tDCS) of the left prefrontal cortex, used as treatment for depression, may induce manic symptoms (36, 37, 93-97). Although conclusions about these brain stimulation effects are based on limited data, our lesion network mapping results are consistent with the existing literature. Combined with prior work showing that lesion network mapping can identify effective brain stimulation targets in other disorders $(3,54,98,99)$ our results might be used to guide future brain stimulation trials for mania (32-35). For example, our results suggest an optimal rTMS target in the right prefrontal cortex (MNI $20,36,52)$, with alternative targets in the right orbitofrontal cortex (MNI 42, 42, -20) and right inferior temporal gyrus (MNI 50, -26, $-16)$, which will require prospective clinical validation.

Our study has several potential limitations. First, our literature cohort is susceptible to selection bias, error in lesion tracing, heterogeneity in imaging modality (CT vs. MRI), and inaccuracies that may result from using a 2-dimensional (2D) lesion to approximate a 3D lesion volume. Similarly, our clinical cohort is limited by small sample size. However, all of these limitations would bias us away from finding a consistent localization and are unlikely to explain the significant results demonstrated here. Moreover, the fact that our results replicate across both cohorts suggest that limitations specific to 1 cohort are unlikely to explain our results. Consistency across our 2 cohorts is also consistent with prior work showing that lesion networks derived from 2D slices are very similar to networks derived from 3D lesions $(20,100)$. Importantly, literature results were replicated in a smaller clinical cohort of MRI-defined 3D lesions, which are not subject to the same limitations as our literature cohort. A second limitation of our study is that lesion network mapping focuses on the spatial component of lesion-induced symptoms, neglecting the temporal evolution of symptoms, brain remodeling in response to injury, and the dynamic process of compensation and/or recovery that could involve brain regions other than those connected to the lesion location. Further work using functional and structural neuroimaging at multiple time points in patients with lesion-induced mania is needed to chart this temporal evolution and assess the role of the regions implicated here in this process. Finally, any implications of these results for primary bipolar disorder must take into consideration that lesion-induced mania could have a different neuroanatomical substrate than primary mania $(74,101,102)$. The alignment of our lesion results with therapeutic effects of brain stimulation in primary mania suggests some overlap, but further work comparing the 2 is needed (for an example see ref. 52). Furthermore, this study focuses only on lesion location, while ignoring potential contributions from genetic loading $(16,86)$, environmental or treatment exposures (87-89), and the use of pharmacologic agents (103-106), with known impact in primary bipolar disorder.

In conclusion, we found that brain lesions associated with mania occur in a variety of different brain locations but share a specific connectivity profile, when compared with lesions not associated with mania. These results lend insight into neuroana- tomic and functional substrates underlying mania and may help define future potential therapeutic targets for this complex neuropsychiatric syndrome.

\section{Methods}

Mania lesion cohort 1: $2 D$ lesions from literature search. Cases of secondary lesional mania with available lesion images published until February 2017 were identified from a literature search according to a predefined protocol (107) following PRISMA guidelines (53). A full list of search terms and of the inclusion and exclusion criteria applied to this study is available in the supplemental material. Cases with traumatic or infectious etiology were excluded because brain damage may extend beyond what is apparent on structural imaging. Two investigators with expertise in psychiatry independently assessed eligibility and extracted clinical and demographic data, with consensus obtained, if necessary, with input from a senior psychiatrist. Two other investigators manually traced lesions onto a standardized brain atlas (MNI) using Medical Imaging Interaction Toolkit (MITK) software (http:// www.mitk.org), with a third investigator independently reviewing each lesion tracing. Because these lesion locations were traced from published images, they are 2D.

Mania lesion cohort 2: 3D lesions from clinical chart review. Clinical cases of secondary lesional mania were identified consecutively by 2 psychiatrists from patients at the Neuropsychiatry Unit of the Psychiatry Department at Centro Hospitalar de Lisboa Ocidental (CHLO), according to protocol approved by the Ethics Committee of CHLO. Eligibility assessment and extraction of clinical and demographic data were performed as described for literature cases. From an initial cohort of 21 patients identified by chart review, 15 were eligible according to the inclusion and exclusion criteria described in the supplemental material, with the remaining excluded due to treatment with corticosteroids and/or antidepressants $(n=3)$ or due to traumatic/ infectious etiology $(n=3)$. One investigator manually traced brain lesions on each MRI using MITK software, with independent review and corrections by another investigator. A third investigator coregistered each traced lesion into MNI space using a composition of 3D rigid and affine transformations, and 3D cubic B-spline transformation, in 3D Slicer software (https://www.slicer.org/). The transformed lesion in atlas space was compared with the original radiologic lesion for verification of accuracy by 3 authors. Because these lesions were traced from the original MRI scans, they are 3D.

Deriving a mania network from brain lesions in the literature. rs-fMRI data from 1000 healthy subjects $(23,24)$ were used to generate a connectivity map for each lesion location, as described previously $(20,73,108)$. Briefly, we computed the correlation between the average time-course of rs-fMRI activity from each lesion location with activity in every other brain voxel, using data from each of the healthy subjects $(73,109)$. Results were combined across the 1000 subjects using a random-effects analysis to generate a lesion network map for each lesion location. To identify connections differing significantly between mania and control lesions, these network maps were statistically compared with lesion network maps derived from control lesion locations, not associated with mania. Literature-derived control lesions associated with nonpsychiatric symptoms, such as aphasia, asterixis, freezing of gait, and poststroke pain $(20,110,111)$, were used according to traces performed previously for the respective publications $(n=79)$. Statistical comparison between the mania-derived 
and control-derived network maps was performed using a voxel-wise permutation-based 2-sample $t$ test implemented within FSL PALM (https://fsl.fmrib.ox.ac.uk/fsl/fslwiki/Randomise/UserGuide), with threshold-free cluster enhancement (TFCE), 2-tailed testing, and family-wise error (FWE) correction options. Two thousand permutations per test and $\alpha$ less than 0.05 were selected. Permutation-based statistics followed by FWE correction was used to reduce the risk of false positives (112), according to best practice guidelines for neuroimaging (113). The resulting voxel statistical map was defined as a "mania lesion network map."

Reliability, validation, reproducibility, and robustness. In order to assess reliability of our tracing methodology, we tested if lesion locations and the resulting mania network map remained stable when literature lesions were traced by a different researcher, independent from the team performing tracing originally. To test lesion location reliability, we compared center of gravity distances obtained from tracing pairs from the same lesion $(N=41$ pairs $)$, to those obtained from tracing of pairs of distinct lesions ( $N=820$ pairs). Because these data did not fit a normal distribution, we performed Wilcoxon's ranksum test (114) (Stata Statistical Software, release 15; StataCorp LLC). To test lesion network mapping reliability, we recomputed the mania lesion network map using the second set of literature tracings. The degree of similarity between mania lesion network maps was computed using Pearson's spatial correlation.

Validation of the literature-based mania lesion network map was tested with data from an independent cohort of lesional mania. Specifically, we tested if the literature-based mania lesion network map could serve as a spatial template to anticipate the probability that a lesion location will result in mania symptoms. We thus tested whether our independent data set of clinical mania lesions $(n=$ 15) intersected the literature-based mania lesion network map more than clinical control lesions. These 3D control lesions were obtained from a cohort of patients with stroke $(n=490)$, that were not selected for any particular symptom (115). For each mania or control lesion, we summed the intensities of voxels in the network map that intersected the lesion location, and then compared sums of voxel intensities between mania and control lesions. Comparisons were performed in a general linear model, controlling for lesion size, bootstrapping the standard error estimates (2000 permutations) (Stata Statistical Software, release 15).

Reproducibility across different cohorts was also tested using data from the clinical cohorts. We computed a second mania lesion network map using the clinical mania lesion cohort $(n=15)$ and the clinical control cohort of stroke lesions $(n=490)$, as described previously for the literature cohort. In the first step a simple conjunction analysis was performed, identifying connections significantly associated with mania in both the literature and clinical network maps. We then performed a single combined analysis in PALM comparing the literature and clinical mania lesion cohorts $(n=56)$ with the literature and clinical control cohorts $(n=569)$. Voxels significantly associated with mania across both cohorts were identified, including dimension (2D vs. 3D) as a covariate. Given extremely robust results, we applied a rigorous voxel-wise FWE correction at $\alpha$ less than $1 \times 10^{-4}$ to highlight only the most significant findings. We identified peak coordinates in this map and ROIs greater than $200 \mathrm{~mm}^{3}$. Coordinates are reported in MNI atlas space and ROIs are labeled using the Harvard-Oxford atlas. Please see Supplemental Table 3 for further details.
The combined (literature + clinical) mania lesion network map was then recomputed multiple times to assess robustness to a variety of different confounds. Importantly, we recomputed the map controlling for lesion size, to ensure that differences in lesion size were not driving the observed connectivity differences between mania and control lesions. We tested whether our results were dependent on the temporal association between lesion onset and emergence of manic symptoms by repeating our analysis using 2 mania subgroups split according to the median time between lesion occurrence and the onset of mania symptoms: either lesions with documented temporal association of 3 years or less $(n=28)$ or lesions with a documented temporal association of more than 3 years, or unknown temporal association $(n=28)$. Analyses to test whether our results were being driven by choice of control lesions were performed in analyses maintaining the same mania lesions but changing control lesions. We replaced the original literature control cohort $(n=79)$ with an alternative literature control cohort consisting of different literature lesions included in previous publications by our group $(n=274)$, independent of whether the symptom profiles might overlap with those of mania and including numerous psychiatric symptoms $(20,25,26,29,54)$. We also replaced our original clinical control cohort $(n=490)$ with an alternate clinical cohort of nonspecific lesions from a different source $(n=135)(116)$. Finally, to ensure results were independent of our inclusion criteria, we recomputed the combined (literature + clinical) mania lesion network map for 4 different mania lesion subgroups: (a) patients with all core mania symptoms listed in DSM $5(n=46)$; (b) patients without a personal or family history of depression, mania, or psychotic disorders $(n=39)$; (c) patients in whom lesion etiology was exclusively ischemic stroke $(n=23)$; and (d) patients satisfying criteria a, b, and c $(n=19)$.

Diagnostic relevance of the mania lesion network. We assessed how a diagnostically challenging case, published after our original literature search (2), could be assessed using the combined mania connectivity network map. Specifically, we intersected all the 6 traceable lesions reported in that case with the combined mania connectivity network map, to obtain the sum of voxel intensities for each separate lesion, as described above for the intersection between clinical cohorts lesions and the literature connectivity network map. We then tested if the sum of voxel intensities of each individual lesion from this case report differed significantly from the distributions of the sums of voxel intensities for the literature mania lesions $(n=41)$ and for the literature control lesions $(n=79)$. Because these data did not fit a normal distribution, these comparisons were performed using a 1-sample Wilcoxon's signed-rank test, with the sum of voxel intensities for the case report lesions as the hypothesized values (Stata Statistical Software, release 15). This analysis returns the probability, i.e., $P$ value, that the sum of voxel intensities for a given lesion is within the distribution of sums of voxel intensities in the comparison cohort (i.e., the mania or control literature cohort) (114). Probabilities for these analyses were reported as percentages instead of decimals (i.e., $P=0.05$ is $5 \%$ ), to facilitate interpretation (114).

Relationship of the mania lesion network to lesion locations causing other symptoms. We explored whether lesion locations associated with other neuropsychiatric symptoms intersected our combined mania lesion network map. Specifically, we examined lesion locations associated with criminality $(n=40)(26)$, delusions $(n=32)(25)$, hemichorea $(n=29)$ (29), alien limb $(n=53)$ (30), akinetic mutism $(n=28)(30)$, auditory hallucinations $(n=28)(20)$, parkinsonism $(n=29)$ (31) and 
visual hallucinations $(n=42)$ (20). Importantly, none of these cohorts were used as controls in the primary analysis used to derive the mania connectivity map. To test whether intersection with the mania lesion map, i.e., sum of voxel intensities, differed across lesion cohorts, we performed a general linear model controlling for lesion size, bootstrapping the standard error estimates (2000 permutations) (Stata Statistical Software, release 15). In planned post hoc analyses, we tested whether lesion intersection with our map was significantly different from zero for each syndrome, as well as differences between specific pairs of syndromes.

Therapeutic relevance of the mania lesion network. To test whether the connectivity profile derived from lesion locations might relate to mania-related clinical responses to high-frequency rTMS (32-37), the connectivity between a priori ROIs representing the left and right DLPFC and the combined mania network map was computed $(4,117)$. The a priori ROIs were the left and right middle frontal gyri selected from the Harvard-Oxford Cortical Structural Atlas, selected to align with the locations of brain stimulation previously reported to induce (left DLPFC) or relieve (right DLPFC) mania symptoms (32-37). Since the atlas parcellations are defined according to the voxel probability of being included in the ROI, we used a 95\% confidence interval threshold (114) to discard voxels that could be located outside of the brain or overlapping with other ROIs. The a priori ROIs thus defined are sufficiently large to account for variability in stimulation site across subjects, occurring due to different methods for target localization and when brain stimulation treatments are performed without neuronavigation (118). We then tested significant differences between left and right DLPFC connectivity to the mania network map, using a paired $t$ test across the connectome $(N=1000)$, as described elsewhere $(4,117)$.

To further explore the therapeutic relevance of the mania network map, we reviewed available literature focusing on rTMS trials for mania treatment, reporting the target localization method. We found 4 trials, all using right-sided targets, 3 of which with target localization using the 5 -cm rule $(34,35,38)$ and 1 using the EEG F4 coordinate (39). Since none used neuronavigation, we extracted the average MNI coordinates for both methods from previous reports: (MNI -41, 16, 54) for the 5-cm method (117) and (MNI 36, 44, 32) for the EEG F4 coordinate (119). After creating a 10-mm spherical ROI (120) centered at each coordinate, we computed the connectivity between these ROIs and the combined mania network map $(4,117)$, and tested significant differences between the 2 using a paired $t$ test across the connectome $(N=1000)(4,117)$.

In another exploratory approach we reviewed the literature to focus on cases of DBS-induced mania reporting target stimulation coordinates. Specifically, we tested if the combined mania network map differentiates DBS targets associated with mania from the average targets, as reported in the literature. We found 5 reports of mania after VC/VS stimulation in patients with obsessive-compulsive disorder (41-44) and 4 reports of mania after STN stimulation in Parkinson's disease patients (47-49), that included stimulation coordinates. Coordinates were mapped into MNI space using Lead-DBS software (www.lead-dbs.org) $(45,121)$. We then averaged the mania-inducing coordinates in the VC/VS (MNI right 10.19, 3.05, -5.69 | left: -9.94, 3.97, -4.13) and STN (MNI right: 11.44, -15.41, -8.57 | left: -11.75 , $-15.94,-8.8)$. Average standard/typical DBS target locations were also defined from previous reports of VC/VS (MNI right: 13, 8, -2 | left: -13, $8,-1)(50)$ and STN (MNI right: 12.58, $-13.41,-5.87$ | left: $-12.58,-13.41$, -5.87) (45) DBS. A 3-mm spherical ROI, approximating previous reports of DBS volume tissue activation (122), was centered at each the mentioned coordinates. Connectivity between each ROI and the combined mania network map was computed $(4,117)$, and significant differences in connectivity between mania-inducing and the respective DBS target were identified using a paired $t$ test across the normative connectome $(N=1000)(4,117)$.

Statistics. As detailed above, the following statistical tests were performed: voxel-wise permutation-based 2-sample $t$ tests to compare mania-derived and control-derived network maps; general linear models to test whether mania lesion map intersections differed across different cohorts, i.e., clinical mania, clinical controls, and other neuropsychiatric symptoms; Wilcoxon's rank-sum and Wilcoxon's signed-rank tests to test reliability of lesion location and the therapeutic relevance of the mania lesion network, respectively; and paired $t$ tests to test differences in connectivity between distinct TMS or DBS targets. Two-tailed $P$ values are reported. Unless otherwise noted, hypothesis tests were interpreted considering a type I error probability of $\alpha$ less than 0.05 .

Study approval. Data collection in the clinical cohort was approved by the ethics committee at Centro Hospitalar de Lisboa Ocidental, Lisbon, Portugal, in accordance with the Declaration of Helsinki, that, considering the retrospective nature of the study, waived the need for written informed consent. Data analysis was approved by the ethics committee at Beth Israel Deaconess Medical Center, Boston, Massachusetts, USA.

\section{Author contributions}

GC, DT, JBBC, AJOM, and MDF conceived and designed the work. GC, DT, JBBC, RR, SS, AV, VCS, NR, OW, and AJOM acquired the data. GC, DT, JH, ALC, LS, AJOM, and MDF analyzed and interpreted data. GC and AV independently assessed eligibility and extracted clinical and demographic data with input from JBBC and/or AJOM. GC and VCS manually traced lesions onto the MNI brain atlas, with SS independently reviewing each lesion tracing. JBBC and AJOM identified clinical cases of secondary lesional mania. RR coregistered each traced lesion into MNI space. GC, JBBC, and SS verified the accuracy of the transformed lesions. DT independently assessed the reliability of our tracing methodology. GC, DT, AJOM, and MDF drafted the manuscript, which was critically revised by the remaining authors for important intellectual content. All authors approved the final version to be published and agree to be accountable for all aspects of the work in ensuring that questions related to the accuracy or integrity of any part of the work are appropriately investigated and resolved. AJOM and MDF had full access to all the data in the study and take responsibility for the integrity of the data and the accuracy of the data analysis. Order of co-first authors was decided by consensus.

\section{Acknowledgments}

GC was supported by a Doctoral Fellowship (SFRH/ BD/130210/2017) from Fundação para a Ciência e Tecnologia (FCT). DT was supported by a DuPont-Warren Fellowship Award from Harvard Medical School. JBBC and AJOM were supported by grant LISBOA-01-0145-FEDER-30302, funded by Portuguese national funds from FCT and cofunded by Fundo Europeu de Desenvolvimento Regional (FEDER), under the Partnership Agreement Lisboa 2020 - Programa Operacional Regional de Lisboa. GC and AJOM were supported by Portuguese national funds 
from FCT through grant PTDC/MED-NEU/31331/2017. ALC was supported by an NIH Ruth L. Kirschstein National Research Service Award (NRSA) Institutional Research Training Grant (T32MH112510) and the Shields Research Grant from the Child Neurology Foundation. MDF was supported by the Sidney R. Baer, Jr. Foundation, the Nancy Lurie Marks Foundation, the Mather's Foundation, and the NIH (grants R01 MH113929, R01 MH115949, and R01 AG060987). None of the funding agencies had a role in the design and conduct of the study, in the collection, management, analysis and interpretation of the data, in the preparation, review or approval of the manuscript, nor in the decision to submit the manuscript for publication.
Address correspondence to: Michael D. Fox, Center for Brain Circuit Therapeutics, Department of Neurology, Psychiatry, and Radiology, Brigham and Women's Hospital, Harvard Medical School, Hale Building 7002P, 60 Fenwood Rd., Boston, Massachusetts 02115, USA. Phone: 617.732.7432; Email: foxmdphd@ gmail.com. Or to: Albino J. Oliveira-Maia, Champalimaud Clinical Centre, Champalimaud Centre for the Unkown, Avenue Brasilia 1400-038, Lisbon, Portugal. Phone: 351.210.480.200 ext. 4065; Email: albino.maia@neuro.fchampalimaud.org.

VCS's present address is: Department of Neuroradiology, Hospital de Braga, Braga, Portugal.
1. Silbersweig D. Integrating models of neurologic and psychiatric disease. JAMA Neurol. 2017;74(7):759-760.

2. Taylor JB, Prager LM, Quijije NV, Schaefer PW. Case 21-2018: A 61-year-old man with grandiosity, impulsivity, and decreased sleep. N EnglJMed. 2018;379(2):182-189.

3. Fox MD. Mapping symptoms to brain networks with the human connectome. $N$ EnglJ Med. 2018;379(23):2237-2245.

4. Padmanabhan JL, et al. A human depression circuit derived from focal brain lesions. Biol Psychiatry. 2019;86(10):749-758.

5. Vernooij MW, et al. Incidental findings on brain MRI in the general population. N Engl JMed. 2007;357(18):1821-1828.

6. Stangeland H, Orgeta V, Bell V. Poststroke psychosis: a systematic review. J Neurol Neurosurg Psychiatry. 2018;89(8):879-885.

7. Santos CO, Caeiro L, Ferro JM, Figueira ML. Mania and stroke: a systematic review. Cerebrovasc Dis. 2011;32(1):11-21.

8. Couleon M, Couleon H, Anne L, Clerc G, Guiraldetrenqualye JP. [Encysted hydroma of the aqueduct of Sylvius with psychomotor excitation syndrome in a person having suffered a mild manic attack 6 years previously. Cure after surgical intervention]. Ann Med Psychol (Paris). 1965;123:627-629.

9. Starkstein SE, et al. Mania after brain injury: neuroradiological and metabolic findings. Ann Neurol.1990;27(6):652-659.

10. Robinson RG, Szetela B. Mood change following left hemispheric brain injury. Ann Neurol. 1981;9(5):447-453.

11. Robinson RG, Kubos KL, Starr LB, Rao K, Price TR. Mood disorders in stroke patients. Importance of location of lesion. Brain. 1984;107(pt 1):81-93.

12. Robinson RG, Boston JD, Starkstein SE, Price TR. Comparison of mania and depression after brain injury: causal factors. Am J Psychiatry. 1988;145(2):172-178.

13. Robinson RG, Starkstein SE. Mood disorders following stroke: new findings and future directions. J Geriatr Psychiatry. 1989;22(1):1-15.

14. Robinson RG, Decarvalho ML, Paradiso S. Post-stroke psychiatric-problems. diagnosis, pathophysiology and drug treatment options. Cns Drugs. 1995;3(6):436-447.

15. Robinson RG. Neuropsychiatric consequences of stroke. Annu Rev Med.1997;48:217-229.

16. Starkstein SE, Pearlson GD, Boston J, Rob- inson RG. Mania after brain injury. A controlled study of causative factors. Arch Neurol. 1987;44(10):1069-1073.

17. Starkstein SE, Boston JD, Robinson RG. Mechanisms of mania after brain injury. 12 case reports and review of the literature. JNerv Ment Dis. 1988;176(2):87-100.

18. Starkstein SE, Fedoroff P, Berthier ML, Robinson RG. Manic-depressive and pure manic states after brain lesions. Biol Psychiatry. 1991;29(2):149-158.

19. Starkstein SE, Robinson RG. Mechanism of disinhibition after brain lesions. J Nerv Ment Dis. 1997;185(2):108-114.

20. Boes AD, et al. Network localization of neurological symptoms from focal brain lesions. Brain. 2015;138(Pt 10):3061-3075.

21. van den Heuvel MP, Hulshoff Pol HE. Exploring the brain network: a review on resting-state fMRI functional connectivity. Eur Neuropsychopharmacol. 2010;20(8):519-534.

22. Fox MD, Greicius M. Clinical applications of resting state functional connectivity. Front Syst Neurosci. 2010;4:19.

23. Yeo BT, et al. The organization of the human cerebral cortex estimated by intrinsic functional connectivity. J Neurophysiol. 2011;106(3):1125-1165.

24. Buckner RL, Roffman JL, Smoller JW. Brain Genomics Superstruct Project (GSP) version 10. Harvard Dataverse. https://doi.org/10.7910/ DVN/25833. Updated August 24, 2014. Accessed July 8, 2020.

25. Darby RR, Laganiere S, Pascual-Leone A, Prasad $S$, Fox MD. Finding the imposter: brain connectivity of lesions causing delusional misidentifications. Brain. 2017;140(2):497-507.

26. Darby RR, Horn A, Cushman F, Fox MD. Lesion network localization of criminal behavior. Proc Natl Acad Sci U S A. 2018;115(3):601-606.

27. Kim YW, Kim HS, An YS. Statistical mapping analysis of brain metabolism in patients with subcortical aphasia after intracerebral hemorrhage: a pilot study of F-18 FDG PET images. Yonsei Med J. 2012;53(1):43-52.

28. Fasano A, Laganiere SE, Lam S, Fox MD. Lesions causing freezing of gait localize to a cerebellar functional network. Ann Neurol. 2017;81(1):129-141.

29. Laganiere S, Boes AD, Fox MD. Network localization of hemichorea-hemiballismus. Neurology. 2016;86(23):2187-2195.

30. Darby RR, Joutsa J, Burke MJ, Fox MD. Lesion network localization of free will. Proc Natl Acad
Sci U S A. 2018;115(42):10792-10797.

31. Joutsa J, Horn A, Hsu J, Fox MD. Localizing parkinsonism based on focal brain lesions. Brain. 2018;141(8):2445-2456.

32. Grisaru N, Chudakov B, Yaroslavsky Y, Belmaker RH. Transcranial magnetic stimulation in mania: a controlled study. Am J Psychiatry. 1998;155(11):1608-1610.

33. Michael N, Erfurth A. Treatment of bipolar mania with right prefrontal rapid transcranial magnetic stimulation. J Affect Disord. 2004;78(3):253-257.

34. Saba G, et al. Repetitive transcranial magnetic stimulation as an add-on therapy in the treatment of mania: a case series of eight patients. Psychiatry Res. 2004;128(2):199-202.

35. Praharaj SK, Ram D, Arora M. Efficacy of high frequency (rapid) suprathreshold repetitive transcranial magnetic stimulation of right prefrontal cortex in bipolar mania: a randomized sham controlled study. J Affect Disord. 2009;117(3):146-150.

36. Xia G, et al. Treatment-emergent mania in unipolar and bipolar depression: focus on repetitive transcranial magnetic stimulation. Int J Neuropsychopharmacol. 2008;11(1):119-130.

37. Ozten E, Sayar GH, Karamustafalioglu O. Hypomanic shift observed during rTMS treatment of patients with unipolar depressive disorder: four case reports. Ann Gen Psychiatry. 2013;12(1):12.

38. Pathak V, Sinha VK, Praharaj SK. Efficacy of adjunctive high frequency repetitive transcranial magnetic stimulation of right prefrontal cortex in adolescent mania: a randomized shamcontrolled study. Clin Psychopharmacol Neurosci. 2015;13(3):245-249.

39. Kaptsan A, Yaroslavsky Y, Applebaum J, Belmaker RH, Grisaru N. Right prefrontal TMS versus sham treatment of mania: a controlled study. Bipolar Disord. 2003;5(1):36-39.

40. Kohl S, et al. Deep brain stimulation for treatmentrefractory obsessive compulsive disorder: a systematic review. BMC Psychiatry. 2014;14:214.

41. Haq IU, et al. A case of mania following deep brain stimulation for obsessive compulsive disorder. Stereotact Funct Neurosurg. 2010;88(5):322-328.

42. Tsai HC, et al. Pilot study of deep brain stimulation in refractory obsessive-compulsive disorder ethnic Chinese patients. Psychiatry Clin Neurosci. 2012;66(4):303-312.

43. Goodman WK, et al. Deep brain stimulation for intractable obsessive compulsive disorder: pilot study using a blinded, staggered-onset design . Biol Psychiatry. 2010;67(6):535-542. 
44. Chang CH, Chen SY, Hsiao YL, Tsai ST, Tsai HC. Hypomania with hypersexuality following bilateral anterior limb stimulation in obsessive-compulsive disorder. J Neurosurg. 2010;112(6):1299-1300.

45. Horn A, Kühn AA, Merkl A, Shih L, Alterman R, Fox M. Probabilistic conversion of neurosurgical DBS electrode coordinates into MNI space. Neuroimage. 2017;150:395-404.

46. Chopra A, et al. Underlying neurobiology and clinical correlates of mania status after subthalamic nucleus deep brain stimulation in Parkinson's disease: a review of the literature. J Neuropsychiatry Clin Neurosci. 2012;24(1):102-110.

47. Mandat TS, Hurwitz T, Honey CR. Hypomania as an adverse effect of subthalamic nucleus stimulation: report of two cases. Acta Neurochir (Wien). 2006;148(8):895-897; discussion 898.

48. Welter ML, et al. Optimal target localization for subthalamic stimulation in patients with Parkinson disease. Neurology. 2014;82(15):1352-1361.

49. Davis RA, et al. Disambiguating the psychiatric sequelae of parkinson's disease, deep brain stimulation, and life events: case report and literature review. Am J Psychiatry. 2017;174(1):11-15.

50. Tyagi $\mathrm{H}$, et al. A randomized trial directly comparing ventral capsule and anteromedial subthalamic nucleus stimulation in obsessivecompulsive disorder: clinical and imaging evidence for dissociable effects. Biol Psychiatry. 2019;85(9):726-734.

51. Kim NY, et al. Lesions causing hallucinations localize to one common brain network [published online October 28, 2019]. Mol Psychiatry. https://doi.org/10.1038/s41380-019-0565-3.

52. Lee I, et al. Diverse pathophysiological processes converge on network disruption in mania. J Affect Disord. 2019;244:115-123.

53. Moher D, Liberati A, Tetzlaff J, Altman DG, PRISMA Group. Preferred reporting items for systematic reviews and meta-analyses: the PRISMA statement. PLoS Med. 2009;6(7):e1000097.

54. Darby RR, Joutsa J, Burke MJ, Fox MD. Lesion network localization of free will. Proc Natl Acad Sci U S A. 2018;115(42):10792-10797.

55. Mimura M, et al. Left frontotemporal hyperperfusion in a patient with post-stroke mania. Psychiatry Res. 2005;139(3):263-267.

56. Julayanont P, Ruthirago D, Alam K, Alderazi YJ. Behavioral disconnection syndrome manifesting as combined mania and visual-auditory hallucinations secondary to isolated right thalamic hemorrhage. J Neuropsychiatry Clin Neurosci. 2017;29(4):401-408.

57. Ross ED, Homan RW, Buck R. Differential hemispheric lateralization of primary and social emotions: Implications for developing a comprehensive neurology for emotions, repression, and the subconscious. Neuropsychiatry Neuropsychol Behav Neurol. 1994;7(1):1-19.

58. Barahona-Corrêa JB, et al. Right-sided brain lesions predominate among patients with lesional mania: evidence from a systematic review and pooled lesion analysis. Transl Psychiatry. 2020;10(1):139.

59. Carran MA, Kohler CG, O'Connor MJ, Bilker WB, Sperling MR. Mania following temporal lobectomy. Neurology. 2003;61(6):770-774.

60. Altshuler LL, et al. Blunted activation in orbitofrontal cortex during mania: a functional mag- netic resonance imaging study. Biol Psychiatry. 2005;58(10):763-769.

61. Blond BN, Fredericks CA, Blumberg HP. Functional neuroanatomy of bipolar disorder: structure, function, and connectivity in an amygdala-anterior paralimbic neural system. Bipolar Disord. 2012;14(4):340-355.

62. Blumberg HP, et al. A functional magnetic resonance imaging study of bipolar disorder: state- and trait-related dysfunction in ventral prefrontal cortices. Arch Gen Psychiatry. 2003;60(6):601-609.

63. Blumberg HP, et al. Rostral and orbital prefrontal cortex dysfunction in the manic state of bipolar disorder. Am J Psychiatry. 1999;156(12):1986-1988.

64. Elliott R, Ogilvie A, Rubinsztein JS, Calderon G, Dolan RJ, Sahakian BJ. Abnormal ventral frontal response during performance of an affective go/ no go task in patients with mania. Biol Psychiatry. 2004;55(12):1163-1170.

65. Rubinsztein JS, et al. Decision-making in mania: a PET study. Brain. 2001;124(pt 12):2550-2563.

66. Strakowski SM, et al. Functional magnetic resonance imaging brain activation in bipolar mania: evidence for disruption of the ventrolateral prefrontal-amygdala emotional pathway. Biol Psychiatry. 2011;69(4):381-388.

67. Clark L, Sahakian BJ. Cognitive neuroscience and brain imaging in bipolar disorder. Dialogues Clin Neurosci. 2008;10(2):153-163.

68. Bora E, Fornito A, Yücel M, Pantelis C. Voxelwise meta-analysis of gray matter abnormalities in bipolar disorder. Biol Psychiatry. 2010;67(11):1097-1105.

69. Abé C, Ekman CJ, Sellgren C, Petrovic P, Ingvar $\mathrm{M}$, Landén $\mathrm{M}$. Manic episodes are related to changes in frontal cortex: a longitudinal neuroimaging study of bipolar disorder 1. Brain . 2015;138(pt 11):3440-3448.

70. Brady RO, et al. Differential brain network activity across mood states in bipolar disorder. JAffect Disord. 2017;207:367-376.

71. Migliorelli R, et al. SPECT findings in patients with primary mania. J Neuropsychiatry Clin Neurosci. 1993;5(4):379-383.

72. O'Connell RA, et al. Single-photon emission computed tomography of the brain in acute mania and schizophrenia. J Neuroimaging. 1995;5(2):101-104.

73. Darby RR, Fox MD. Reply: Capgras syndrome: neuroanatomical assessment of brain MRI findings in an adolescent patient. Brain. 2017;140(7):e44.

74. Hibar DP, et al. Cortical abnormalities in bipolar disorder: an MRI analysis of 6503 individuals from the ENIGMA Bipolar Disorder Working Group. Mol Psychiatry. 2018;23(4):932-942.

75. Schaefer PW, Copen WA, Lev MH, Gonzalez RG. Diffusion-weighted imaging in acute stroke. Magn Reson Imaging Clin N Am. 2006;14(2):141-168.

76. Birenbaum D, Bancroft LW, Felsberg GJ. Imaging in acute stroke. West JEmerg Med. 2011;12(1):67-76.

77. Satzer D, Bond DJ. Mania secondary to focal brain lesions: implications for understanding the functional neuroanatomy of bipolar disorder. Bipolar Disord. 2016;18(3):205-220.

78. Jorge RE, Robinson RG, Moser D, Tateno A, Crespo-Facorro B, Arndt S. Major depression following traumatic brain injury. Arch Gen Psychiatry. 2004;61(1):42-50.

79. Hackett ML, Yapa C, Parag V, Anderson CS. Frequency of depression after stroke: a systematic review of observational studies. Stroke. 2005;36(6):1330-1340.

80. Huang J, et al. Association between depression and brain tumor: a systematic review and meta-analysis. Oncotarget. 2017;8(55):94932-94943.

81. Merikangas KR, et al. Lifetime and 12-month prevalence of bipolar spectrum disorder in the National Comorbidity Survey replication. Arch Gen Psychiatry. 2007;64(5):543-552.

82. Bromet E, et al. Cross-national epidemiology of DSM-IV major depressive episode. BMC Med. 2011;9:90.

83. Gagnon LM, Patten SB. Major depression and its association with long-term medical conditions. Can J Psychiatry. 2002;47(2):149-152.

84. Ferguson MA, et al. A human memory circuit derived from brain lesions causing amnesia. Nat Commun. 2019;10(1):3497.

85. Cohen AL, Soussand L, Corrow SL, Martinaud O, Barton JJS, Fox MD. Looking beyond the face area: lesion network mapping of prosopagnosia. Brain. 2019;142(12):3975-3990.

86. Lieberman JA, First MB. Psychotic disorders. N Engl JMed. 2018;379(3):270-280.

87. Ulla $\mathrm{M}$, et al. Manic behaviour induced by deep-brain stimulation in Parkinson's disease: evidence of substantia nigra implication? J Neurol Neurosurg Psychiatry. 2006;77(12):1363-1366.

88. Ulla M, et al. Contact dependent reproducible hypomania induced by deep brain stimulation in Parkinson's disease: clinical, anatomical and functional imaging study. J Neurol Neurosurg Psychiatry. 2011;82(6):607-614.

89. Mosley PE, Marsh R, Perry A, Coyne T, Silburn P. Persistence of mania after cessation of stimulation following subthalamic deep brain stimulation. J Neuropsychiatry Clin Neurosci. 2018;30(3):246-249.

90. Christopher PP, McCabe PJ, Fisher WH. Prevalence of involvement in the criminal justice system during severe mania and associated symptomatology. Psychiatr Serv. 2012;63(1):33-39.

91. Dunayevich E, Keck PE. Prevalence and description of psychotic features in bipolar mania. Curr Psychiatry Rep. 2000;2(4):286-290.

92. Gold AK, et al. Clinical applications of transcranial magnetic stimulation in bipolar disorder. Brain Behav. 2019;9(10):e01419.

93. Gupta A, Adnan M. Hypomania risk in noninvasive brain stimulation. Cureus. 2018;10(2):e2204.

94. Brunoni AR, Moffa AH, Sampaio-Júnior B, Gálvez V, Loo CK. Treatment-emergent mania/ hypomania during antidepressant treatment with transcranial direct current stimulation (tDCS): A systematic review and meta-analysis. Brain Stimul. 2017;10(2):260-262.

95. Berlow YA, Zandvakili A, Carpenter LL, Philip NS. Transcranial direct current stimulation for unipolar depression and risk of treatment emergent mania: An updated meta-analysis. Brain Stimul. 2019;12(4):1066-1068.

96. Brunoni AR, et al. Trial of electrical directcurrent therapy versus escitalopram for depression. N Engl J Med. 2017;376(26):2523-2533. 
97. Pavlova EL, et al. Transcranial direct current stimulation of 20-and 30-minutes combined with sertraline for the treatment of depression. Prog Neuropsychopharmacol Biol Psychiatry. 2018;82:31-38.

98. Joutsa J, et al. Identifying therapeutic targets from spontaneous beneficial brain lesions. Ann Neurol. 2018;84(1):153-157.

99. Casper RC. Nutrients, neurodevelopment, and mood. Curr Psychiatry Rep. 2004;6(6):425-429.

100.Sutterer MJ, Bruss J, Boes AD, Voss MW, Bechara A, Tranel D. Canceled connections: Lesionderived network mapping helps explain differences in performance on a complex decisionmaking task. Cortex. 2016;78:31-43.

101. Chai XJ, et al. Abnormal medial prefrontal cortex resting-state connectivity in bipolar disorder and schizophrenia. Neuropsychopharmacology. 2011;36(10):2009-2017.

102. Phillips ML, Swartz HA. A critical appraisal of neuroimaging studies of bipolar disorder: toward a new conceptualization of underlying neural circuitry and a road map for future research. Am J Psychiatry. 2014;171(8):829-843.

103. Goldberg JF, Truman CJ. Antidepressantinduced mania: an overview of current controversies. Bipolar Disord. 2003;5(6):407-420.

104.Peet M, Peters S. Drug-induced mania. Drug Saf. 1995;12(2):146-153.

105. Maier F, et al. Hypomania and mania related to dopamine replacement therapy in Parkinson's disease. Parkinsonism Relat Disord. 2014;20(4):421-427.
106. Brown ES, Chandler PA. Mood and cognitive changes during systemic corticosteroid therapy. Prim Care Companion J Clin Psychiatry. 2001;3(1):17-21.

107. Cotovio G, et al. Systematic review and meta-analysis on secondary bipolar disorder following an acute brain insult. https://www. crd.york.ac.uk/prospero/display_record. php?ID=CRD42016053675]. Updated December 10, 2016. Accessed July 8, 2020.

108. Joutsa J, Horn A, Hsu J, Fox MD. Localizing parkinsonism based on focal brain lesions. Brain. 2018;141(8):2445-2456.

109. Horn A, et al. Connectivity predicts deep brain stimulation outcome in Parkinson disease. Ann Neurol. 2017;82(1):67-78.

110. Kim YW, Kim HS, An YS. Statistical mapping analysis of brain metabolism in patients with subcortical aphasia after intracerebral hemorrhage: a pilot study of F-18 FDG PET images. Yonsei Med J. 2012;53(1):43-52.

111. Fasano A, Laganiere SE, Lam S, Fox MD. Lesions causing freezing of gait localize to a cerebellar functional network. Ann Neurol. 2017;81(1):129-141.

112. Eklund A, Nichols TE, Knutsson H. Cluster failure: Why fMRI inferences for spatial extent have inflated false-positive rates. Proc Natl Acad Sci U S A. 2016;113(28):7900-7905.

113. Poldrack RA, et al. Scanning the horizon: towards transparent and reproducible neuroimaging research. Nat Rev Neurosci. 2017;18(2):115-126.

114. Rosner B. Fundamentals of Biostatistics. 8th ed. Cengage Learning; 2016.
115. Wu O, et al. Role of acute lesion topography in initial ischemic stroke severity and long-term functional outcomes. Stroke. 2015;46(9):2438-2444.

116. Corbetta M, et al. Common behavioral clusters and subcortical anatomy in stroke. Neuron. 2015;85(5):927-941.

117. Fox MD, Buckner RL, White MP, Greicius MD, Pascual-Leone A. Efficacy of transcranial magnetic stimulation targets for depression is related to intrinsic functional connectivity with the subgenual cingulate. Biol Psychiatry. 2012;72(7):595-603.

118. Weigand A, et al. Prospective validation that subgenual connectivity predicts antidepressant efficacy of transcranial magnetic stimulation sites. Biol Psychiatry. 2018;84(1):28-37.

119. Cutini S, Scatturin P, Zorzi M. A new method based on ICBM152 head surface for probe placement in multichannel fNIRS. Neuroimage. 2011;54(2):919-927.

120. Cash RFH, Zalesky A, Thomson RH, Tian Y, Cocchi L, Fitzgerald PB. Subgenual functional connectivity predicts antidepressant treatment response to transcranial magnetic stimulation: independent validation and evaluation of personalization. Biol Psychiatry. 2019;86(2):e5-e7.

121. Horn A, Kühn AA. Lead-DBS: a toolbox for deep brain stimulation electrode localizations and visualizations. Neuroimage. 2015;107:127-135.

122. Maks CB, Butson CR, Walter BL, Vitek JL, McIntyre CC. Deep brain stimulation activation volumes and their association with neurophysiological mapping and therapeutic outcomes. J Neurol Neurosurg Psychiatry. 2009;80(6):659-666. 